\title{
Osteoma osteoide en hueso ganchoso
}

\section{Simón Pérez ${ }^{(1)}$, R. Hernández Ramajo ${ }^{(2)}$, V. García Virto ${ }^{(2)}$, M. Á Martín Ferrero ${ }^{(3)}$}

\author{
Hospital Clínico Universitario de Valladolid \\ (1) Médico Adjunto de Traumatología y Cirugía Ortopédica. Profesor Asociado de Traumatología Y \\ CIRUGÍA ORTOPÉDICA UNIVERSIDAD DE VALLADOLID \\ (2) Médico Residente de Traumatología y Cirugía Ortopédica \\ (3) Jefe de Sección de La Unidad de Mano. Profesor Titular de Traumatología y Cirugía Ortopédica \\ UNIVERSIDAD DE VALLADOLID
}

\author{
Correspondencia: \\ Dra. Clarisa Simón Pérez \\ Hospital Clínico Universitario de Valladolid \\ Servicio CO y $\mathrm{T}$ \\ Avda Ramón y Cajal, s/n \\ 47005 Valladolid \\ Teléf. 983420000 ext 276 \\ e-mail: simonclarisa@yahoo.es
}

El osteoma osteoide es un tumor óseo benigno poco frecuente en la muñeca. Se caracteriza por la aparición de dolor inespecífico sin relación con actividades, que empeora por la noche y mejora con la administración de ácido acetilsalicílico. Su diagnóstico se basa en la clínica y en las pruebas se imagen, fundamentalmente en la TAC y la RM. El tratamiento consiste en la exéresis quirúrgica.

Presentamos un caso clínico de un osteoma osteoide en el ganchoso junto a una revisión de la literatura.

Palabras clave: osteoma osteiode, muñeca, hueso ganchoso, nidus.
The osteoid osteoma is a benign bone tumor that occurs infrequently in the wrist. It is characterized by the appearance of nonspecific wrist pain unrelated to activity that worsens at night and improves with administration of acetylsalicylic acid. Diagnosis is based on clinical and image evidence is primarily in CT and MRI. Treatment consists of surgical excision.

We report a case of osteoid osteoma in the hamate with a review of the literature.

Key words: osteoid osteoma, wrist, hamate, nidus.

\section{INTRODUCCIÓN}

$\mathrm{L}$ os tumores óseos benignos de la mano son poco frecuentes, aunque existen algunas series descritas a lo largo de los años ${ }^{1}$. La mayoría de los pacientes son diagnosticados cuando acuden a consulta por otras razones como: síndromes del túnel del carpo, artritis, dolor inespecífico de muñeca... ${ }^{1}$.

El osteoma osteoide es un tumor óseo benigno descrito por Jaffe en $1935^{2}$, aunque existe una publicación previa de Bergstrand de $1930^{3}$. Este tumor representa el $3 \%$ de los tumores óseos, constituyendo el $10 \%$ de los tumores óseos benignos. Ha sido descrito en todos los huesos del esqueleto salvo en el cráneo y el esternón, localizándose principalmente a nivel de los huesos largos como tibia y fémur $(50-60 \%)^{2}$.

Solamente el $10 \%$ de los casos de osteomas osteoides afectan la mano. La localización en los huesos del carpo es del $2 \%{ }^{4}$ siendo la más frecuente en el escafoides y el hueso grande. 

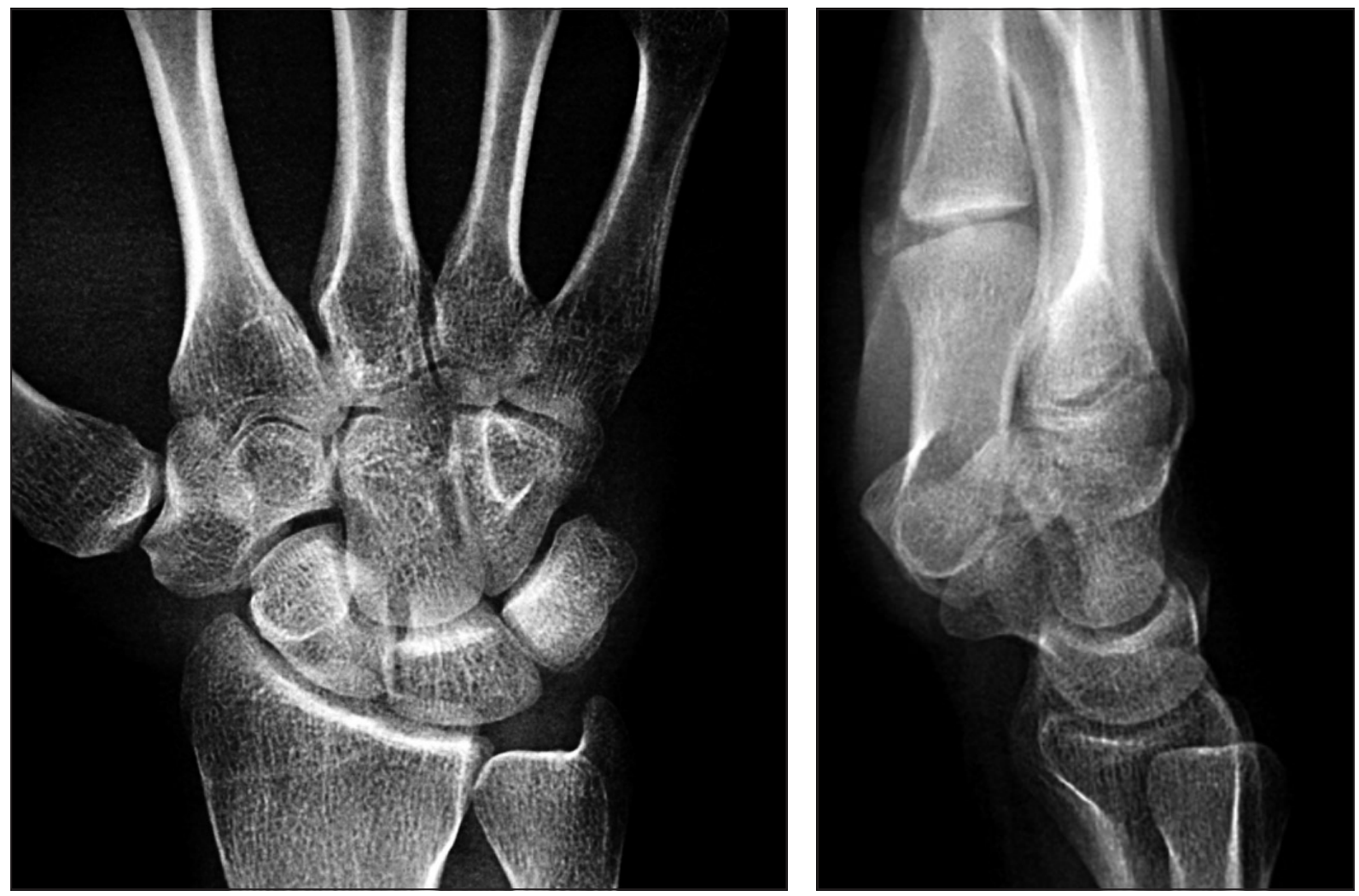

Figura 1. Estudio radiográfico de la muñeca.

Se manifiesta clínicamente por dolor nocturno, que remite tras la administración de ácido acetilsalicílico, e infamación local. En el estudio radiográfico de observa una pequeña lesión radiolucente, patognomónica, denominada nidus.

Existen pocos casos descritos en la literatura de osteomas osteoides localizados en el hueso ganchoso.

\section{CASO CLÍNICO}

Varón de 26 años de edad, que consultó por presentar dolor en la muñeca derecha de meses de evolución, sin antecedente traumático ni aumento del dolor con la actividad deportiva ni laboral. El dolor era nocturno, despertaba al paciente por la noche, y no cedía con la ingesta de analgésicos habituales.

En la exploración física no se apreciaba inflamación, edema ni deformidad evidente, a la palpación aparecía dolor a la presión de la zona cubital de la articulación mediocarpiana. La movilidad estaba conservada en todos sus grados y no era dolorosa.

En el estudio de imagen, las radiografías simples de la muñeca en proyección anteroposterior y lateral eran normales (Figura 1).

En el estudio mediante RM de muñeca derecha se observaba un aumento generalizado de la señal en secuencias T2, y en la superficie dorsal, del tercio medio del hueso ganchoso, una lesión cortical de aproximadamente $3 \mathrm{~mm}$ de menor señal (Figura 2).

En la TAC de muñeca se evidenciaba una imagen de $3 \mathrm{~mm}$ en el hueso ganchoso de localización dorsal y perióstica compatible con osteoma osteoide (Figura 3).

La gammagrafía ósea en dos fases con $\mathrm{Tc}^{99 \mathrm{~m}}$ MDP mostraba, en fase vascular una intensa hiperemia a nivel del hueso ganchoso y en el estudio tardío, una imagen redonda hiperactiva en la misma localización, compatible con un proceso muy vascularizado con intensa actividad osteogénica en hueso ganchoso (Figura 4). 


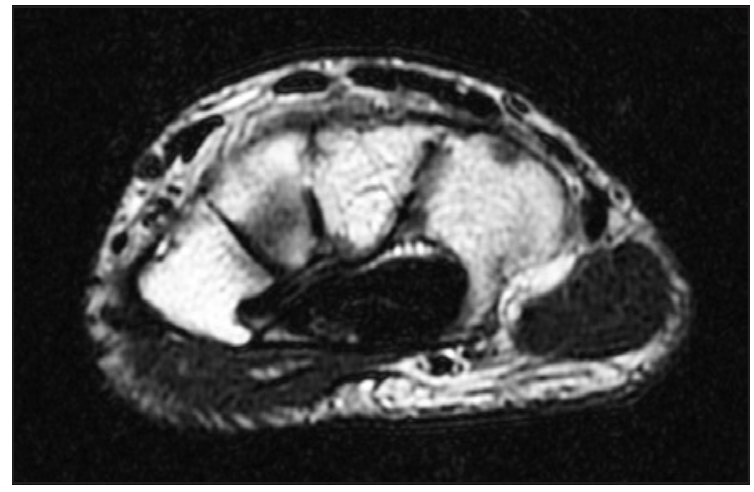

Figura 2. Estudio mediante RM.

Tras los estudios realizados y una vez confirmada la lesión se decidió intervención quirúrgica mediante la exéresis completa de la lesión con márgenes de seguridad.

Para ello mediante escopia se localizó el hueso ganchoso, y con una incisión cutánea transversa de $0,5 \mathrm{~cm}$, tras proteger las partes blandas, se colocó una trefina de $0,5 \mathrm{~cm}$ de diámetro alrededor de la misma extrayendo en bloque la lesión y curetando los márgenes.

A los 3 meses de la intervención quirúrgica persistía dolor en la zona cicatricial que se solucionó con una sesión de radiofrecuencia.

En el momento actual el paciente está asintomático con buena función de la muñeca y mano.

\section{DISCUSIÓN}

El osteoma osteoide localizado en la mano y muñeca es infrecuente. Afecta principalmente a

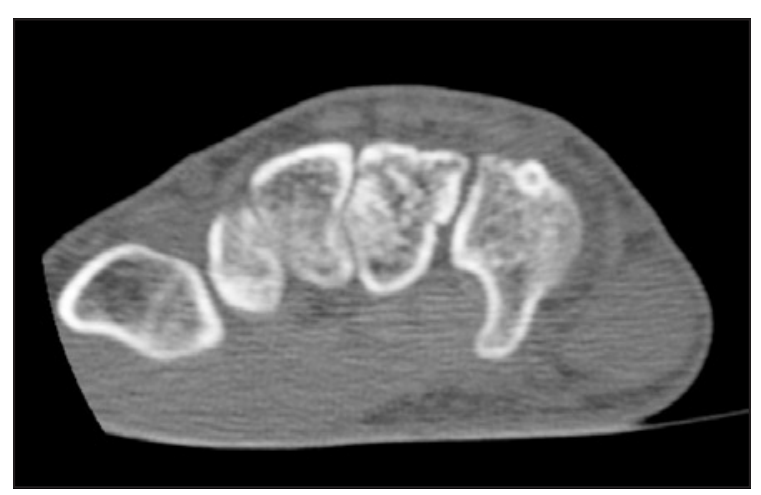

Figura 3. Estudio mediante TAC.

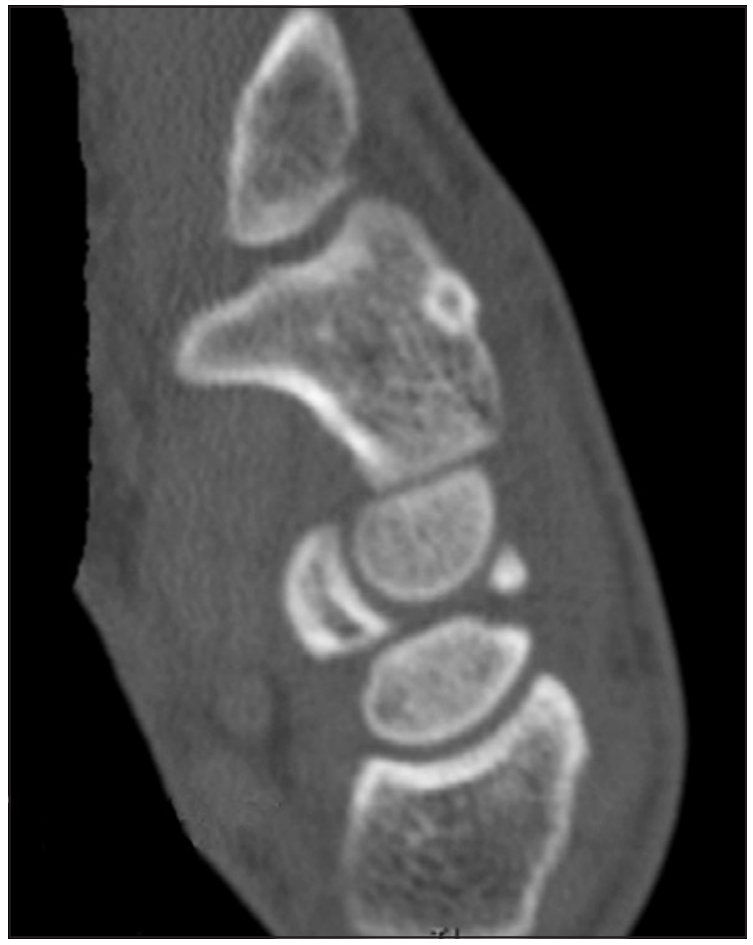

Figura 4. Gammagrafía ósea.

pacientes con edades comprendidas entre los 15 y 45 años y sobre todo de sexo masculino ${ }^{4}$.

La clínica, cuando se localiza a nivel de los huesos del carpo, es dolor inespecífico de la muñeca, sin relación con la actividad física ni antecedente traumático previo, que empeora por la noche y mejora tras la administración de ácido acetilsalicílico.

En la exploración se puede apreciar la presencia de inflamación de los tejidos blandos, junto con rubor y calor de la piel circundante, lo cual se debe a la naturaleza vascular del tumor que ocasiona tensión y edema. ${ }^{4}$ Otro hallazgo frecuente, como describe Lluch $^{5}$, es la presencia de una sinovitis articular y tendinosa en la vecindad del tumor que contribuye a la inflamación local y al dolor.

A pesar de esta clínica su presentación en ocasiones es insidiosa, y suele existir una historia previa de molestias localizadas a nivel de la muñeca durante el ejercicio deportivo o en trabajos manuales ${ }^{6}$. Esto lleva a que a veces el diagnóstico se retrase hasta varios meses desde el comienzo de los síntomas ${ }^{7-9}$.

En el diagnóstico por imagen la presencia del típico nidus con imagen radiolucente rodeada de 
esclerosis puede faltar debido a la superposición de los huesos del carpo y al pequeño tamaño de estos huesos ${ }^{10}$. Por esa razón para su diagnóstico se emplean la TAC y la RM. La TAC es la prueba más específica y nos muestra la lesión con más precisión $^{11,12}$. Por otra parte la RM es la prueba más sensible para el diagnóstico ya que muestra el edema óseo y de los tejidos blandos en las secuencias en T2 10,13. Para autores como Assoun et al. existe una correlación negativa entre el empleo de antiinflamatorios y las imágenes de edema obtenidas por $\mathrm{RM}^{11}$.

Con la RM, en algunos casos, se aprecia una importante sinovitis peri-lesional que, como en un caso de la serie de Irisarri ${ }^{14}$, no tiene por qué afectar a los tendones próximos.

La gammagrafía ósea con Tecnecio 99m también puede ser necesaria para el diagnóstico ya que va a mostrar una imagen de captación a nivel de la lesión lo que indica la naturaleza vascular del proceso ${ }^{15}$.

El tratamiento del osteoma osteoide se basa en la exéresis quirúrgica del nidus con una tasa de recurrencia muy baja, que se debe principalmente a la extirpación incompleta del mismo $^{16-18}$. Si la resección tumoral es extensa suele ser necesario rellenar el defecto cavitario con sustitutos óseos o con injerto óseo autólogo. Las imágenes previas de TAC y RM son de gran ayuda para la planificación preoperatoria del abordaje quirúrgico; así, cuando el tumor se localiza en zona dorsal de la muñeca se em- plea un abordaje dorsal, que de forma rápida permite llegar bien a la lesión y proceder a su extirpación y curetaje, a la vez que permite hacer sinovectomía de lo tejidos adyacentes ${ }^{6}$. Por otro lado si la lesión se localiza en cara palmar hay que hacer una adecuada separación de vasos, nervios y tendones previos a la exéresis del tumor ${ }^{6,19}$.

Otra alternativa terapéutica para el tratamiento del osteoma osteoide, sobre todo en tumores en los que su abordaje quirúrgico es difícil, es la termocoagulación percutánea guiada por $\mathrm{TAC}^{20,21}$; aunque esta técnica no está exenta de complicaciones ya que puede causar necrosis de los tejidos en un área de aproximadamente $1 \mathrm{~cm}$ cuadrado alrededor de la lesión.

\section{CONCLUSIONES}

El osteoma osteoide es un tumor benigno que cuando se localiza en la muñeca provoca dolor de características inespecíficas, y que para su diagnóstico son necesarias pruebas de imagen como la TAC y la RM. Su tratamiento se basa principalmente en la extirpación quirúrgica de la lesión aunque en la actualidad está aumentando el empleo de la ablación por radiofrecuencia con unos buenos resultados.

El objetivo del presente trabajo es presentar un caso a nivel del hueso ganchoso, lugar donde no es frecuente su localización.

\section{BIBLIOGRAFÍA}

1. Murray $P$, Berger R, MD, Inwards C. Primary neoplasms of the carpal bones? J Hand Surg Am, 1999; 24: 1008-13.

2. Jaffe L. Osteoid-osteoma. A bening osteoblastic tumor composed of osteoid and atypical bone. Arch Surg, 1935; 31, 709-28.

3. Bergstrand $H$. Ober eine eigenartige, wahrscheinlich bisher nicht heschreibene osteoblastische Rrankheit in den langenknochen der hand und des Fusses. Acta Radiol, 1930; 11, 596-612.
4. Thomsen L, Dumontier C. Osteoid osteoma of the pisiform. Chir Main, 2011; 30: 76-9.

5. Lluch A. Osteomas osteoides de la muñeca. Revisión de seis casos. Rev Esp Cir Mano, 1991; 41: 23-8.

6. Laffossea JM, Tricoirea JL, Cantagrelb A, Wagnerc A, Pugeta J. Osteoid osteoma of the carpal bones. Two case reports. Joint Bone Spine, 2006; 73: 560-3.

7. Tricoire JL, Duport M, Puget J, Mazieres B, Chiron P, Utheza G. Osteome osteoide du trape- zoide. Ann Chir Main, 1991; 10: 175-7.

8. Girard J, Becquet E, Limousin M, Chantelot C, Fontaine C. Osteome osteoide de l'os trapezoide: a propos d'un cas et revue de la litterature. Chir Main, 2005; 24: 35-8.

9. Kreitner KF, Low R, Mayer A. Unusual manifestation of an osteoid osteoma of the capitate. Eur Radiol, 1999; 9: 1098100.

10. De Smet L, Brys P, Fabry G, Baert A. An unusual localisation and presentation of an osteo- 
id osteoma. Acta Orthop Belg, 1997; 63: 128-31.

11. Assoun J, Richardi G, Railhac JJ, Baunin C, Fajadet P, Giron $\mathrm{J}$, et al. Osteoid osteoma: MR imaging versus $\mathrm{CT}$. Radiology, 1994; 191: 217-23.

12. De Smet L, Fabry G. Osteoid osteoma of the hand and carpus: peculiar presentations and imaging. Acta Orthop Belg, 1995; 61: 113-6.

13. Schindler A, Hodler J, Michel BA, Bruehlmann P. Osteoid osteoma of the capitate. Arthritis Rheum, 2002; 46: 2808-10.

14. Irisarri C, Capomassi M. Tumores óseos benignos. En: Tumores de la Mano, Cap. 7. Madrid:
Ed. Méd. Panamericana, 2009: 65-84.

15. Smith FW, Gilday DL. Scintigraphic appearances of osteoid osteoma. Radiology, 1980; 137: 191-5.

16. Rosenfeld K, Bora FW Jr, Lane JM. Osteoid osteoma of the hamate: a case report and review of the literature. J Bone Joint Surg Am, 1973; 55: 1085-7.

17. Murray PM, Berger RA, Inwards CY. Primary neoplasms of the carpal bones. J Hand Surg Am, 1999; 24: 1008-13.

18. Arora J, McLauchlan J, Munro N. Recurrent osteoid osteoma of the lunate: a case report and review of the literature. Hand Surg, 2003; 8: 239-42.

19. Herndon JH, Eaton RG, Littler JW. Carpal-tunnel syndrome. An unusual presentation of osteoid osteoma of the capitate. $J$ Bone Joint Surg Am, 1974; 56: 1715-8.

20. Donahue F, Ahmad A, Mnaymneh W, Pevsner N. Osteoid osteoma. Computed tomography guided percutaneous excision. Clin Orthop, 1999; 366: 191-6.

21. Rosenthal DI, Alexander A, Rosenberg AE, Springfield DS. Ablation of osteoid osteomas with a percutaneously placed electrode: a new procedure. Radiology, 1992; 183: 29-33. 